\title{
SYNTHESIS AND PHARMACOLOGICAL EVALUATION OF SOME NEW 1,2,4-TRIAZOLO QUINAZOLINE DERIVATIVES
}

\author{
Mohamed H, El-Shershaby ${ }^{1}$, Ashraf H. Bayoumi ${ }^{1}$, Adel Ghiaty ${ }^{1}$, Hamada S. Abulkhair ${ }^{1,2 *}$ \\ ${ }^{1}$ Department of Pharmaceutical Organic Chemistry, Faculty of Pharmacy, Al-Azhar \\ University, Nasr City 11884, Cairo, Egypt \\ ${ }^{2}$ Department of Pharmaceutical Chemistry, Faculty of Pharmacy, Horus University - \\ Egypt, International Coastal Road, 34518, New Damietta, Egypt \\ *Corresponding author: hamadaorganic@azhar.edu.eg
}

\begin{abstract}
Cancer is a major public health problem in all countries and remains as one of the top leading causes of death worldwide. Herein we report the synthesis of novel series of triazoloquinazolines as potential anticancer agents. Structures of this set of compounds were confirmed by different spectral data including IR, ${ }^{1} \mathrm{H} \mathrm{NMR},{ }^{13} \mathrm{C} \mathrm{NMR}$, Mass spectra, and elemental analyses. All the newly synthesized compounds were evaluated for their antiproliferative activity against four human cancer cell lines namely; Hepatocellular carcinoma (HePG-2), Mammary gland breast cancer (MCF-7), Human prostate cancer (PC3) and Colorectal carcinoma (HCT-116)). Results of cytotoxicity evaluation showed that the synthesized compounds displayed moderate cytotoxic activity against the selected cancer cell lines. Compound 9 showed the highest anti-proliferative effect followed by compound 7 against colorectal carcinoma cell line (HCT-116) with $\mathrm{IC}_{50}$ values of 17.35 and $27.05 \mu \mathrm{M}$ respectively. As well, both compounds showed moderate activity against hepatocellular carcinoma (HePG2) with $\mathrm{IC}_{50}$ values of 29.47 and $39.41 \mu \mathrm{M}$ respectively.
\end{abstract}

Keywords: Triazoloquinazolines, Anticancer activity, cytotoxicity evaluation, doxorubicin. 


\section{Introduction}

Quinazoline derivatives are biologically important heterocycles and are known to possess a variety of biological activities such as antitumor (Fedorov et al. 2014), antimicrobial (Sun et al. 2011; Sun, Wei, et al. 2010), anti-inflammatory (Sun, Hu, et al. 2010), antihistaminic (Awadallah, El-Eraky, and Saleh 2012), antidepressant (Olmo et al. 2006), anticonvulsant (Abulkhair et al. 2016) and antihypertensive (Holló et al. 2014). Quinazoline scaffold is in the core structure of many commercially available anticancer drugs like, Gefitinib (1) (Eldehna et al. 2017) Erlotinib (2) (Gaber et al. 2018), and Lapatinib (3) (Figure 1) which were approved by FDA in the last decade for the treatment certain types of cancers (wood E. R. et al. 2004). On the other hand, Triazole moieties were reported to possess antitumor activity (Ezzat et al. 2020; Turky, Bayoumi, et al. 2020; Turky, Sherbiny, et al. 2020). Recently, two research articles (Alesawy et al. 2020; Ewes et al. 2020) reported the 1,2,4-triazolo[4,3-c] quinazolines as a new class of anticancer agents with potential inhibitory effects of EGFR-TK, and topoisomerase II. These latter studies documented the EGFR inhibition activity of 4 with $\mathrm{IC}_{50}$ value of 0.69 to $1.8 \mu \mathrm{M}$. Cell cycle study of MCF-7 cancer cell showed that the same compound could arrest cell cycle at G2/M phase and induced the apoptotic cell death. Considering the above-mentioned facts and in continuation of our recent works (El-Shershaby et al. 2020; Hannoun et al. 2020) to develop a new potential molecules with potent bioactivity, molecular hybridization between triazole and quinazoline as effective antitumor moieties was carried out. All the synthesized compounds were evaluated for their in vitro anticancer activity against four cancer cell lines namely; hepatocellular carcinoma (HePG-2), mammary gland breast cancer (MCF-7), human prostate cancer (PC3) and colorectal carcinoma (HCT-116).
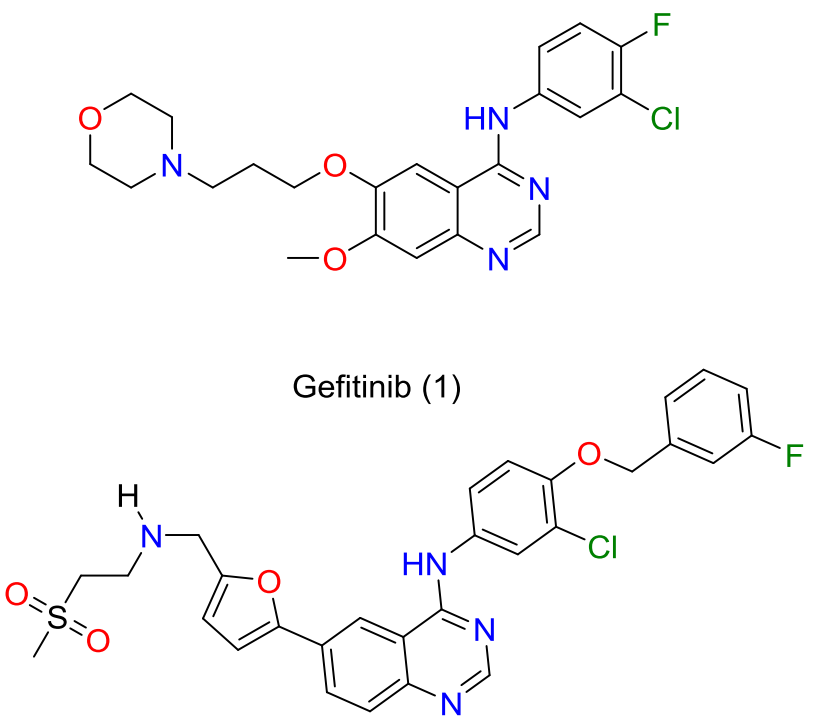

Lapatinib (3)

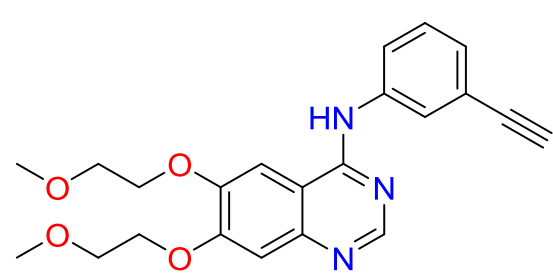

Erlotinib (2)

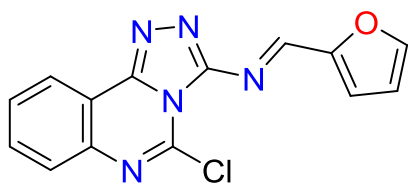

4

Figure 1: Structures of some anticancer agents containing quinazoline and triazoloquinazoline nuclei 


\section{Results and discussion}

Chemistry: The general route for the synthesis of starting triazoloquinazolines is presented in Scheme 1. The $o$-ureidobenzoic acids was prepared from the corresponding anthranilic acid $\mathbf{1}$ and potassium cyanate (Asif 2014). The ureido acids are then easily cyclized to the respective quinazoline-2,4(1H,3H)-dione 2 by heating with acid or alkali. Treating of quinazoline-2,4(1H,3H)-dione 2 with Phosphorus oxychloride (Fujino et al. 2001) in the presence of diisopropylethylamine (DIPEA) or $N, N$-dimethylaniline to provide 2,4dichloroquinazoline 3. 2-Chloroquinazolin-4-yl)hydrazine $\mathbf{4}$ was prepared by adding hydrazine hydrate (Abul-Khair et al. 2013; Abulkhair et al. 2020) dropwise to alcoholic solution of 2,4-dichloro quinazoline 3 at $0-5^{\circ} \mathrm{C}$. The resulting mixture was stirred for $0.5 \mathrm{~h}$ below $10^{\circ} \mathrm{C}$ then at room temperature for additional 2 hours. Compound 4 was heated in water bath with acetic anhydride $(40 \mathrm{~mL})$ for 3-4 hours then leaved to cool to obtain 3methyl[1,2,4]triazolo[4,3-c]quinazolin-5(6H)-one (5) in a reasonable good yield. treating the latter with $p$-methylbenzenesulfonyl chloride in DMF for $4 \mathrm{hr}$ afforded compound $\mathbf{6}$.

6-cinnamoyl-3-methyl[1,2,4]triazolo[4,3-c]quinazolin-5(6H)-one (8) synthesized by reaction of $\mathbf{5}$ with cinnamoyl chloride in the presence of potassium iodide as a catalyst and dry DMF as a solvent. Reaction of compounds $\mathbf{5}$ with the appropriate the chloroesters in DMF and heating for 3-5 hr afforded the desired alkyl 2-(3-substituted-5-oxo-[1,2,4] triazolo[4,3-c]quinazolin-6(5H)-yl) acetate derivatives $8 \mathbf{a}$ and $\mathbf{8 b}$. Compound $\mathbf{5}$ was also allowed to react with 2-chloro- $N$-phenylacetamide in DMF and in the presence of sodium acetate. this alternative route successfully afforded the final product $\mathbf{9}$ in a satisfactory yield and reasonable purities. 

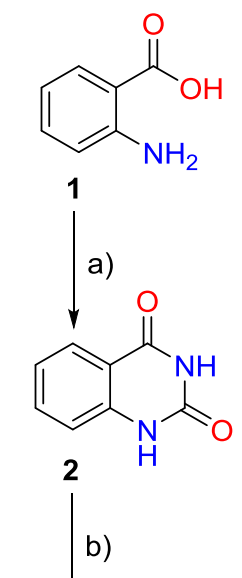<smiles>Clc1nc(Cl)c2ccccc2n1</smiles><smiles></smiles>

4 5

Reagent and conditions:

a) $\mathrm{KCNO}$, glacial $\mathrm{ACOH}, \mathrm{NAOH} / \mathrm{HCL}$, b) $\mathrm{POCL}_{3}, \mathrm{PCL}_{5}, 110^{\circ} \mathrm{C}, 5 \mathrm{hr}$. c) $\mathrm{NH}_{2} \mathrm{NH}_{2} \cdot \mathrm{H}_{2} \mathrm{O}$, Methanol, TEA, rt, $2 \mathrm{hr}$. d) Acetic anhydride, $110{ }^{\circ} \mathrm{C}, 4 \mathrm{hr}$ e) Methylbenzenesulfonyl chloride, $\mathrm{DMF}, \mathrm{K}_{2} \mathrm{CO}_{3}, \mathrm{KI}$, $4 \mathrm{hr}$.

f) Cinnamoyl chloride, $\mathrm{DMF}, \mathrm{K}_{2} \mathrm{CO}_{3}, \mathrm{KI}, 4 \mathrm{hr}$.

g) chloroacetate ester, DMF, $3 \mathrm{hr}$.

h) Chloro- $N$-phenyl acetamide , $\mathrm{K}_{2} \mathrm{CO}_{3}$, DMF, $3 \mathrm{hr}$. e)<smiles>Cc1ccc(S(=O)(=O)n2c(=O)n3c(C)nnc3c3ccccc32)cc1</smiles>

6

f)<smiles>Cc1nnc2c3ccccc3n(C(=O)/C=C/c3ccccc3)c(=O)n12</smiles>

g)<smiles>[R]OC(=O)Cn1c(=O)n2c(C)nnc2c2ccccc21</smiles>

h)<smiles>Cc1nnc2c3ccccc3n(CC(=O)Nc3ccccc3)c(=O)n12</smiles>

Scheme 1: Synthetic routes for preparation of target triazoloquinazolines. 
Anticancer evaluation: Anticancer activities of the synthesized compounds were assessed on four cancer cell lines namely; hepatocellular carcinoma (HePG-2), mammary gland breast cancer (MCF-7), human prostate cancer (PC3) and colorectal carcinoma (HCT-116) using MTT assay (Mosmann 1983). Doxorubicin was used as a reference anticancer agent. The results of preliminary anticancer evaluation are shown in Table 1. The results of cytotoxicity evaluation showed that the majority of the synthesized compounds displayed moderate cytotoxic activities against the selected cell lines. Compound 9showed the highest inhibitory effect followed by compound 7 against colorectal carcinoma cell line (HCT-116) with $\mathrm{IC}_{50}$ values of 17.35 and $27.05 \mu \mathrm{M}$ respectively. The same two compounds also showed moderate activity against hepatocellular carcinoma (HePG2) with $\mathrm{IC}_{50}$ values of 29.74 and $39.41 \mu \mathrm{M}$ respectively. Compounds $(\mathbf{5 , 6}$ and $\mathbf{8 a , b})$ displayed relatively lower inhibitory potencies than all other compounds.

Table 1: In vitro anticancer activity of the new triazoloquinazolines.

\begin{tabular}{|c|c|c|c|c|}
\hline \multicolumn{5}{|c|}{ In vitro Cytotoxicity $\mathbf{I C}_{\mathbf{5 0}}(\boldsymbol{\mu M})^{\bullet}$} \\
\hline Compound No. & HePG2 & MCF-7 & PC3 & HCT-116 \\
\hline $\mathbf{5}$ & $88.66 \pm 4.8$ & $94.47 \pm 5.2$ & $>100$ & $65.46 \pm 4.1$ \\
\hline $\mathbf{6}$ & $71.90 \pm 3.9$ & $73.80 \pm 3.8$ & $48.29 \pm 2.9$ & $42.48 \pm 3.2$ \\
\hline $\mathbf{7}$ & $39.41 \pm 2.7$ & $45.20 \pm 2.9$ & $53.10 \pm 3.2$ & $27.05 \pm 2.2$ \\
\hline $\mathbf{8 a}$ & $91.45 \pm 4.9$ & $>100$ & $>100$ & $74.18 \pm 4.4$ \\
\hline $\mathbf{8 b}$ & $67.04 \pm 3.7$ & $90.27 \pm 5.0$ & $83.86 \pm 4.6$ & $57.13 \pm 3.8$ \\
\hline $\mathbf{9}$ & $29.47 \pm 2.3$ & $26.39 \pm 2.5$ & $32.75 \pm 2.5$ & $17.35 \pm 1.5$ \\
\hline Doxorubicin & $4.50 \pm 0.2$ & $4.17 \pm 0.2$ & $8.87 \pm 0.6$ & $5.23 \pm 0.3$ \\
\hline
\end{tabular}

$\mathbf{I C}_{\mathbf{5 0}}(\boldsymbol{\mu M}): 1-10$ (very strong). $11-20$ (strong). $21-50$ (moderate). $51-100$ (weak) and above 100 (non-cytotoxic).

\section{Experimental Section}

General: All melting points were determined using capillary tubes with a Stuart SMP30 apparatus and are uncorrected. ${ }^{1} \mathrm{H}$ NMR spectra were recorded on Bruker- $400-\mathrm{MHz}$ spectrophotometer using DMSO- $\mathrm{d}_{6}$ as a solvent and TMS as internal reference. ${ }^{13} \mathrm{C} \mathrm{NMR}$ spectra were recorded on Bruker-100-MHz spectrophotometer using DMSO- $\mathrm{d}_{6}$ as a solvent and TMS as internal reference. Chemical shifts were recorded in $\delta$ ppm downfield the TMS signal. Mass spectra were recorded on Hewlett Packard 5988 spectrometer. Elemental analyses were performed on $\mathrm{CHN}$ analyzer. All spectral measurements have been performed at the Regional Center for Mycology \& Biotechnology, Al-Azhar University, Cairo, Egypt. Anticancer evaluation was carried out at the Holding Company for Biological Products and Vaccines, Egypt (Vacsera). 


\section{Synthesis of (3-methyl -[1,2,4]triazolo[4,3-c]quinazolin-5(6H)-one (5).}

A mixture of 2-chloro-4-hydrazinylquinazoline $4(4.0 \mathrm{~g}, 0.020 \mathrm{~mol})$ and acetic anhydride $(40 \mathrm{ml})$ was heated in water bath for $4 \mathrm{~h}$, and leaved to cool overnight. The obtained solid was filtered and washed with water and dried, crystallized with methanol to give the target compound $\mathbf{5}$ as a yellow crystal. The structure of compound $\mathbf{5}$ was confirmed by both elemental and spectral analysis.

Yellow solid, Yield: 92\%; m.p. $259-261^{\circ} \mathrm{C}$. IR $(\mathrm{KBr}) \mathrm{cm}^{-1}: 3186(\mathrm{NH}), 3062(\mathrm{CH}$ aromatic), $2985\left(\mathrm{CH}\right.$ aliphatic), $1743(\mathrm{C}=\mathrm{O}), 1627(\mathrm{C}=\mathrm{N}), 1597\left(\mathrm{C}=\mathrm{C}\right.$ aromatic). ${ }^{1} \mathrm{H}$ NMR (DMSO-d6) $\delta$ ppm: 12.19 (S, 1H, NH), 8.06-8.08 (d, $J=8.4 \mathrm{~Hz}, 1 \mathrm{H}, \mathrm{H}-10$ of quinazoline), $7.64-7.66(\mathrm{dd}, J=7.6 \mathrm{~Hz}, 1 \mathrm{H}, \mathrm{H}-8$ of quinazoline), $7.39-7.41(\mathrm{~d}, J=8.4 \mathrm{~Hz}, 1 \mathrm{H}, \mathrm{H}-7$ of quinazoline), 7.35-7.37 (dd, $J=8.4 \mathrm{~Hz}, 1 \mathrm{H}, \mathrm{H}-9$ of quinazoline), $2.48\left(\mathrm{~S}, 3 \mathrm{H}, \mathrm{CH}_{3}\right)$. MS $(\mathrm{m} / \mathrm{z}): 200\left(\mathrm{C}_{1} \cdot \mathrm{H}_{\wedge} \mathrm{N}_{4} \mathrm{O}, 88.71 \%, \mathrm{M}^{+}\right), 199\left(\mathrm{C}_{10} \mathrm{H}_{7} \mathrm{~N}_{4} \mathrm{O}, 15.67 \%\right) .{ }^{13} \mathrm{C}$ NMR (DMSO-d6) $\delta$ ppm: 163.36, 153.15, 144.13, 137.32, 132.98, 124.38, 123.92, 116.42, 110.57, and 14.53. Anal. Calc. for: $\left(\mathrm{C}_{10} \mathrm{H}_{8} \mathrm{~N}_{4} \mathrm{O}\right)(\mathrm{M} . \mathrm{W} .=200)$ : C, 59.99; H, 4.03; N, $27.99 \%$; Found: C, $61.89 ; \mathrm{H}, 4.85 ; \mathrm{N}, 25.98 \%$.

\section{Synthesis of 3-methyl-6-tosyl-[1,2,4]triazolo[4,3-c]quinazolin-5(6H)-one (6).}

To a mixture of compound 5 (0.200 g, 0.001 mole) and $\mathrm{K}_{2} \mathrm{CO} 3$ in dry DMF (20 $\mathrm{mL})$, 4-methylbenzenesulfonyl chloride $(0.190 \mathrm{~g}, 0.001$ mole) was added in presence of catalytic amount of $\mathrm{KI}$ and heated under refluxing using water bath for $4 \mathrm{~h}$. After cooling to room temperature, the reaction mixture was poured onto ice-water $(100 \mathrm{ml})$ and stirred for $1 \mathrm{~h}$. The obtained solid was filtered and crystallized from ethanol.

Brown solid, Yield: 79\%; m.p. 276-278 ${ }^{\circ} \mathrm{C}$. IR (KBr) $\mathrm{cm}^{-1}$ : 3031 (CH aromatic), 2924 (CH aliphatic), $1734(\mathrm{C}=\mathrm{O}), 1595$ (C=C aromatic). ${ }^{1} \mathrm{H}$ NMR (DMSO- $\left.d 6\right) \delta$ ppm: 7.93 (d, $1 \mathrm{H}, J=8 \mathrm{~Hz}, \mathrm{H}-10$ of quinazoline), 7.52 (dd, $2 \mathrm{H},, J=8 \mathrm{~Hz}, \mathrm{H}-2, \mathrm{H}-3$ of tosyl), 7.43 (dd, $1 \mathrm{H},, J=7.6 \mathrm{~Hz}, \mathrm{H}-8$ of quinazoline), 7.27 (d, $1 \mathrm{H},, J=8.4 \mathrm{~Hz}, \mathrm{H}-7$ of quinazoline), 7.14 (dd, $2 \mathrm{H}, J=8 \mathrm{~Hz}, 5 \mathrm{H}, 6 \mathrm{H}$ of tosyl) $7.02(\mathrm{dd}, 1 \mathrm{H}, J=8.4 \mathrm{~Hz}, \mathrm{H}-9$ of quinazoline), 2.44 (S, $\left.3 \mathrm{H}, \mathrm{CH}_{3}\right), 2.28\left(\mathrm{~S}, 3 \mathrm{H}, \mathrm{CH}_{3}\right.$ of tosyl). $\mathrm{MS}(\mathrm{m} / \mathrm{z}): 354\left(\mathrm{C}_{17} \mathrm{H}_{14} \mathrm{~N}_{4} \mathrm{O}_{3} \mathrm{~S}, 58.36 \%, \mathrm{M}^{+}\right), 339\left(\mathrm{C}_{16}\right.$ $\left.\mathrm{H}_{11} \mathrm{~N}_{4} \mathrm{O}_{3} \mathrm{~S}, 10.15 \%\right), 324\left(\mathrm{C}_{15} \mathrm{H}_{8} \mathrm{~N}_{4} \mathrm{O}_{3} \mathrm{~S}, 10.15 \%\right) .{ }^{13} \mathrm{C}$ NMR (DMSO-d6) $\delta$ ppm: 167.46 , $161.09,153.35,149.38,146.99,145.16,138.69$, 131.22, 129.94, 128.68, 127.97, 126.00, 123.41, 122.99, 120.23, 21.30 and 14.72. Anal. Calc. for: $\left(\mathrm{C}_{17} \mathrm{H}_{14} \mathrm{~N}_{4} \mathrm{O}_{3} \mathrm{~S}\right)(\mathrm{M} . \mathrm{W} .=354)$ : $\mathrm{C}$, 57.62; H, 3.98; N, $15.81 \%$; Found: C, 57.85; H, 4.12; N, $16.07 \%$.

\section{Synthesis of 6-cinnamoyl-3-methyl-[1,2,4]triazolo[4,3-c]quinazolin-5(6H)-one (7).}

To a mixture of Compound $5\left(0.200 \mathrm{~g}, 0.001\right.$ mole) and $\mathrm{K}_{2} \mathrm{CO} 3$ in dry DMF (20 $\mathrm{mL})$, Cinnamoyl chloride $(0.166 \mathrm{~g}, 0.001$ mole $)$ was added in presence of catalytic amount of KI and heated under refluxing using water bath for $6 \mathrm{~h}$. After cooling to room temperature, the reaction mixture was poured onto ice-water $(100 \mathrm{ml})$ and stirred for $1 \mathrm{~h}$. The obtained solid was filtered and crystallized from ethanol. 
Brown solid, Yield: 76\%; m.p. $281-283^{\circ} \mathrm{C}$. IR (KBr) $\mathrm{cm}^{-1}: 3065$ (CH aromatic), 2925 (CH aliphatic), 1689 (C=O), 1654 (C=O aromatic). ${ }^{1} \mathrm{H}$ NMR (DMSO- $\left.d 6\right) \delta$ ppm: 8.46 (d, $1 \mathrm{H}, J=8 \mathrm{~Hz}, \mathrm{H}-10$ of quinazoline), $7.46(\mathrm{dd}, 1 \mathrm{H}, J=7.6 \mathrm{~Hz}, \mathrm{H}-8$ of quinazoline), 7.34 (d, 2H, H-2, H-6 of phenyl), 7.32 (d, $1 \mathrm{H}, J=8.4 \mathrm{~Hz}, \mathrm{H}-7$ of quinazoline), 7.31 (d, 2H, H-3, $\mathrm{H}-5$ of phenyl), 7.26 (dd, $1 \mathrm{H},, J=8.4 \mathrm{~Hz}, \mathrm{H}-9$ of quinazoline), 7.00 (t, 1H, H-4 of phenyl), $6.32\left(\mathrm{~S}, 2 \mathrm{H}, \mathrm{H}\right.$ of alkene). $\mathrm{MS}(\mathrm{m} / z): 330\left(\mathrm{C}_{19} \mathrm{H}_{14} \mathrm{~N}_{4} \mathrm{O}_{2}, 55.58 \%, \mathrm{M}^{+}\right), 298\left(\mathrm{C}_{19} \mathrm{H}_{14} \mathrm{~N}_{4}, 12.9\right.$ $\%), 283\left(\mathrm{C}_{18} \mathrm{H}_{11} \mathrm{~N}_{4}, 26.96 \%\right)$. Anal. Calc. for: $\left(\mathrm{C}_{19} \mathrm{H}_{14} \mathrm{~N}_{4} \mathrm{O}_{2}\right)$ (M.W. = 330): C, 69.08; $\mathrm{H}$, 4.27; N, $16.96 \%$; Found: C, 69.31; H, 4.50; N, $17.23 \%$.

Synthesis of alkyl 2-(3-methyl -5-oxo-[1,2,4]triazolo[4,3-c]quinazolin-6(5H)-yl) acetate derivatives $(\mathbf{8 a}, 8 \mathbf{b})$.

\section{General procedure:}

To a mixture of Compound $5\left(0.200 \mathrm{~g}, 0.001\right.$ mole) and $\mathrm{K}_{2} \mathrm{CO} 3$ in dry DMF (20 $\mathrm{mL})$, an appropriate Methyl chloroacetate or Ethyl chloroacetate $(0.002$ mole) was added and heated using water bath for $3 \mathrm{~h}$. After cooling to room temperature, the reaction mixture was poured onto ice-water $(100 \mathrm{ml})$ and stirred for $1 \mathrm{~h}$. The obtained solid was filtered and crystallized from ethanol.

\section{Synthesis of Methyl 2-(3-methyl -5-oxo-[1,2,4]triazolo[4,3-c]quinazolin-6(5H)-yl) acetate (8a).}

Yellowish solid, Yield: 86\%; m.p. 233-235 ${ }^{\circ} \mathrm{C}$. IR (KBr) cm ${ }^{-1}: 3047$ (CH aromatic), 2997 (CH aliphatic), 1716 (C=O), 1620 (C=C aromatic). ${ }^{1} \mathrm{H}$ NMR (DMSO- $\left.d 6\right) \delta$ ppm: 8.10 (d, $1 \mathrm{H}, J=8 \mathrm{~Hz}, \mathrm{H}-10$ of quinazoline), 7.50 (dd, $1 \mathrm{H}, J=7.6 \mathrm{~Hz}, \mathrm{H}-8$ of quinazoline), 7.42 (d, $1 \mathrm{H}, J=8 \mathrm{~Hz}, \mathrm{H}-7$ of quinazoline), $7.38(\mathrm{dd}, 1 \mathrm{H}, J=8.4 \mathrm{~Hz}, \mathrm{H}-9$ of quinazoline), 5.21 $(\mathrm{S}, 2 \mathrm{H}, \mathrm{N}-\mathrm{CH} 2), 3.74\left(\mathrm{~S}, 3 \mathrm{H}, \mathrm{OCH}_{3}\right), 2.45\left(\mathrm{~S}, 3 \mathrm{H}, \mathrm{CH}_{3}\right) . \mathrm{MS}(\mathrm{m} / z): 272\left(\mathrm{C}_{13} \mathrm{H}_{12} \mathrm{~N}_{4} \mathrm{O}_{3}\right), 213$ $\left(\mathrm{C}_{11} \mathrm{H}_{9} \mathrm{~N}_{4} \mathrm{O}, 26.96 \%\right) .{ }^{13} \mathrm{C}$ NMR (DMSO-d6) $\delta \mathrm{ppm}: 168.76,153.19,152.39,144.71$, 137.64, 133.73, 125.20, 123.29, 116.46, 110.60, 53.06, 45.54 and 14.54. Anal. Calc. for: $\left(\mathrm{C}_{13} \mathrm{H}_{12} \mathrm{~N}_{4} \mathrm{O}_{3}\right)(\mathrm{M} . W .=272): \mathrm{C}, 57.35 ; \mathrm{H}, 4.44 ; \mathrm{N}, 20.58 \%$; Found: C, 57.49; H, 4.63; N, $20.39 \%$.

Synthesis of Ethyl 2-(3-methyl -5-oxo-[1,2,4]triazolo[4,3-c]quinazolin-6(5H)-yl) acetate $(\mathbf{8 b})$.

Yellowish white solid, Yield: $79 \%$; m.p. $238-240^{\circ} \mathrm{C}$. IR $(\mathrm{KBr}) \mathrm{cm}^{-1}: 3059(\mathrm{CH}$ aromatic), 2993 (CH aliphatic), $1728(\mathrm{C}=\mathrm{O}), 1624$ ( $\mathrm{C}=\mathrm{C}$ aromatic). ${ }^{1} \mathrm{H}$ NMR (DMSO-d6) $\delta$ ppm: $8.13(\mathrm{~d}, 1 \mathrm{H}, J=8 \mathrm{~Hz}, \mathrm{H}-10$ of quinazoline), 7.51 (dd, $1 \mathrm{H}, J=7.6 \mathrm{~Hz}, \mathrm{H}-8$ of quinazoline), $7.42(\mathrm{~d}, 1 \mathrm{H}, J=8.4 \mathrm{~Hz}, \mathrm{H}-7$ of quinazoline), $7.38(\mathrm{dd}, 1 \mathrm{H}, J=8.4 \mathrm{~Hz}, \mathrm{H}-9 \mathrm{of}$ quinazoline), $5.20(\mathrm{~S}, 2 \mathrm{H}, \mathrm{N}-\mathrm{CH} 2), 4.20\left(\mathrm{q}, 2 \mathrm{H}, J=8.0 \mathrm{~Hz}, \mathrm{OCH}_{2}\right), 2.51(\mathrm{t}, 3 \mathrm{H}, J=7.6 \mathrm{~Hz}$, $\left.\mathrm{OCH}_{2}-\mathrm{CH}_{3}\right), 1.24\left(\mathrm{~s}, 3 \mathrm{H}, \mathrm{CH}_{3}\right)$. MS $(\mathrm{m} / \mathrm{z}): 286\left(\mathrm{C}_{14} \mathrm{H}_{14} \mathrm{~N}_{4} \mathrm{O}_{3}, 15.79 \%, \mathrm{M}^{+}\right), 213\left(\mathrm{C}_{11} \mathrm{H}_{9}\right.$ $\mathrm{N}_{4} \mathrm{O}, 20.44 \%$ ). ${ }^{13} \mathrm{C}$ NMR (DMSO-d6) $\delta$ ppm: 168.25, 164.22, 152.39, 144.72, 137.66, 133.74, 125.21, 124.80, 116.04, 111.05, 110.59, 62.02, 45.63, 14.47 and 14.46. Anal. Calc. 
for: $\left(\mathrm{C}_{14} \mathrm{H}_{14} \mathrm{~N}_{4} \mathrm{O}_{3}\right)(\mathrm{M} . \mathrm{W} .=286)$ : C, 58.74; H, 4.93; N, $19.57 \%$; Found: C, 58.53; H, 4.79; $\mathrm{N}, 19.81 \%$.

Synthesis of 2-(3-methyl-5-oxo-[1,2,4]triazolo[4,3-c]quinazolin-6(5H)-yl)-Nphenylacetamide (9).

To a mixture of Compound 5 ( $0.200 \mathrm{~g}, 0.001$ mole) and $\mathrm{K}_{2} \mathrm{CO} 3$ in dry DMF (20 $\mathrm{mL})$, 2-chloro- $N$ - phenyl acetamide $(0.169 \mathrm{~g}, 0.001$ mole) was added and heated using water bath for 3 hours. After cooling to room temperature, the reaction mixture was poured onto ice-water $(100 \mathrm{ml})$ and stirred for $1 \mathrm{~h}$. The obtained solid was filtered and crystallized from Ethanol.

Brown solid, Yield: $66 \%$; m.p. $253-255^{\circ} \mathrm{C}$. IR $(\mathrm{KBr}) \mathrm{cm}^{-1}$ : $3258(\mathrm{NH}), 3062(\mathrm{CH}$ aromatic), 2978 ( $\mathrm{CH}$ aliphatic), $1687(\mathrm{C}=\mathrm{O}), 1592\left(\mathrm{C}=\mathrm{C}\right.$ aromatic). ${ }^{1} \mathrm{H}$ NMR (DMSO-d6) $\delta$ ppm: $10.21\left(\mathrm{~S}, 1 \mathrm{H}, \mathrm{NH}, \mathrm{D}_{2} \mathrm{O}\right.$ exchanged), $7.90(\mathrm{~d}, 1 \mathrm{H}, J=8.4 \mathrm{~Hz}, \mathrm{H}-10$ of quinazoline), $7.88(\mathrm{~d}, 1 \mathrm{H}, J=8 \mathrm{~Hz}, \mathrm{H}-7$ of quinazoline), $7.62(\mathrm{~d}, 2 \mathrm{H}$, phenyl), $7.42(\mathrm{dd}, 1 \mathrm{H}, J=7.6 \mathrm{~Hz}$, H-8 of quinazoline), 7.38 (t, $2 \mathrm{H}$, phenyl), 7.08 (dd, $1 \mathrm{H}, J=8.4 \mathrm{~Hz}, \mathrm{H}-9$ of quinazoline), 7.04 (t, $1 \mathrm{H}$, phenyl), $4.14\left(\mathrm{~S}, 2 \mathrm{H}, \mathrm{N}-\mathrm{CH}_{2}\right), 2.45\left(\mathrm{~S}, 3 \mathrm{H}, \mathrm{CH}_{3}\right)$. MS $(\mathrm{m} / \mathrm{z}): 333\left(\mathrm{C}_{18} \mathrm{H}_{15} \mathrm{~N}_{5} \mathrm{O}_{2}\right.$, $\left.\mathrm{M}^{+}\right), 168\left(\mathrm{C}_{9} \mathrm{H}_{4} \mathrm{~N}_{4}, 10.43 \%\right) .{ }^{13} \mathrm{C}$ NMR (DMSO-d6) $\delta \mathrm{ppm}: 168.94,156.94,144.43,139.34$, $138.85,134.19,129.34,129.30,123.76,121.06,119.63,115.91,57.80$, and 13.20. Anal. Calc. for: $\left(\mathrm{C}_{18} \mathrm{H}_{15} \mathrm{~N}_{5} \mathrm{O}_{2}\right)(\mathrm{M} . \mathrm{W} .=333)$ : C, 64.86; H, 4.54; N, $21.01 \%$; Found: C, 65.09; H, $4.63 ; \mathrm{N}, 20.89 \%$.

Anticancer activity: All the synthesized compounds were subjected to MTT proliferation assay to investigate their in-vitro cytotoxic activity. Hepatocellular carcinoma (HePG-2), mammary gland breast cancer (MCF-7), Human prostate cancer (PC3) and colorectal carcinoma (HCT-116) cell lines was chosen for investigation.

\section{Conclusion}

A series of triazoloquinazoline derivatives was synthesized and evaluated for their anticancer activity against four human cancer cell lines (Hepatocellular carcinoma (HePG2), mammary gland breast cancer (MCF-7), Human prostate cancer (PC3) and Colorectal carcinoma (HCT-116)). The results of anticancer evaluation showed that most of the synthesized compounds displayed moderate cytotoxic activities against the selected cell lines. Compound $\mathbf{9}$ showed the highest inhibitory effect followed by compound $\mathbf{7}$ against hepatocellular carcinoma and colorectal carcinoma cell lines.

\section{REFERENCES}

Abul-Khair, Hamada, Salwa Elmeligie, Ashraf Bayoumi, Adel Ghiaty, Ahmed ElMorsy, and Memy H. Hassan. 2013. "Synthesis and Evaluation of Some New $(1,2,4)$ Triazolo(4,3-a)Quinoxalin-4(5H)-One Derivatives as AMPA Receptor Antagonists." Journal of Heterocyclic Chemistry (50):1202-8. 
Abulkhair, Hamada S., Kamal M. El Gamal, Khaled El Adl, and Mohamed F Fadl. 2016. "Molecular Docking, Synthesis and Biological Evaluation of Some Novel 2Substituted-3-Allyl-4(3H)-Quinazolinone Derivatives as Anticonvulsant Agents." Medicinal Chemistry 6(9):593-603.

Abulkhair, Hamada S., Abdallah Turky, Adel Ghiaty, Hany E. A. Ahmed, and Ashraf H. Bayoumi. 2020. "Novel Triazolophthalazine-Hydrazone Hybrids as Potential PCAF Inhibitors: Design, Synthesis, in Vitro Anticancer Evaluation, Apoptosis, and Molecular Docking Studies.” Bioorganic Chemistry 100:103899.

Alesawy, Mohamed S., Ahmed A. Al- Karmalawy, Eslam B. Elkaeed, Mohamed Alswah, Ahmed Belal, Mohammed S. Taghour, and Ibrahim H. Eissa. 2020. "Design and Discovery of New 1,2,4-triazolo[4,3- c ]Quinazolines as Potential DNA Intercalators and Topoisomerase II Inhibitors.” Archiv Der Pharmazie. e2000237.

Asif, Mohammad. 2014. "Chemical Characteristics, Synthetic Methods, and Biological Potential of Quinazoline and Quinazolinone Derivatives." International Journal of Medicinal Chemistry 2014:1-27.

Awadallah, Fadi M., Wafaa I. El-Eraky, and Dalia O. Saleh. 2012. "Synthesis, Vasorelaxant Activity, and Molecular Modeling Study of Some New Phthalazine Derivatives." European Journal of Medicinal Chemistry 52:14-21.

El-Shershaby, Mohamed H., Kamal M. El-Gamal, Ashraf H. Bayoumi, Khaled ElAdl, Hany E. A. Ahmed, and Hamada S. Abulkhair. 2020. "Synthesis, Antimicrobial Evaluation, DNA Gyrase Inhibition, and in Silico Pharmacokinetic Studies of Novel Quinoline Derivatives.” Archiv Der Pharmazie e2000277.

Eldehna, Wagdy M., Hadia Almahli, Ghada H. Al-Ansary, Hazem A. Ghabbour, Mohamed H. Aly, Omnia E. Ismael, Abdullah Al-Dhfyan, and Hatem A. AbdelAziz. 2017. "Synthesis and in Vitro Anti-Proliferative Activity of Some Novel Isatins Conjugated with Quinazoline/Phthalazine Hydrazines against Triple-Negative Breast Cancer MDA-MB-231 Cells as Apoptosis-Inducing Agents." Journal of Enzyme Inhibition and Medicinal Chemistry 32(1):600-613.

Ewes, Wafaa A., Mohammad A. Elmorsy, Shahenda M. El-Messery, and Magda N. A. Nasr. 2020. "Synthesis, Biological Evaluation and Molecular Modeling Study of [1,2,4]-Triazolo[4,3-c]Quinazolines: New Class of EGFR-TK Inhibitors.” Bioorganic \& Medicinal Chemistry 28(7):115373.

Ezzat, Hany G., Ashraf H. Bayoumi, Farag F. Sherbiny, Ahmed M. El-Morsy, Adel Ghiaty, Mohamed Alswah, and Hamada S. Abulkhair. 2020. "Design, Synthesis, and Molecular Docking Studies of New [1,2,4]Triazolo[4,3-a]Quinoxaline Derivatives as Potential A2B Receptor Antagonists." Molecular Diversity,1-16. 
Fedorov, Oleg, Hannah Lingard, Chris Wells, Octovia P. Monteiro, Sarah Picaud, Tracy Keates, Clarence Yapp, Martin Philpott, Sarah J. Martin, Ildiko Felletar, Brian D. Marsden, Panagis Filippakopoulos, Susanne Müller, Stefan Knapp, and Paul E. Brennan. 2014. “[1,2,4]Triazolo[4,3- a ]Phthalazines: Inhibitors of Diverse Bromodomains." Journal of Medicinal Chemistry 57(2):462-76.

Fujino, Kenji, Hitoshi Takami, Toshiyuki Atsumi, Takehiro Ogasa, Shin-ichiro Mohri, and Masaji Kasai. 2001. "Development of a Practical Synthetic Route of a PDE V Inhibitor KF31327.” Organic Process Research \& Development 5(4):426-33.

Gaber, Ahmed A., Ashraf H. Bayoumi, Ahmed M. El-morsy, Farag F. Sherbiny, Ahmed B. M. Mehany, and Ibrahim H. Eissa. 2018. "Design, Synthesis and Anticancer Evaluation of 1H-Pyrazolo[3,4-d]Pyrimidine Derivatives as Potent EGFRWTand EGFRT790Minhibitors and Apoptosis Inducers." Bioorganic Chemistry 80(June):375-95.

Hannoun, M. H., M. Hagras, A. Kotb, A. A. M. M. El-Attar, and H. S. Abulkhair. 2020. "Synthesis and Antibacterial Evaluation of a Novel Library of 2-(Thiazol-5-Y1)1,3,4-Oxadiazole Derivatives against Methicillin-Resistant Staphylococcus Aureus (MRSA)." Bioorganic Chemistry 94.

Holló, Berta, József Magyari, Vukosava Živković-Radovanović, Gordana Vučković, Zoran D. Tomić, Imre Miklós Szilágyi, György Pokol, and Katalin Mészáros Szécsényi. 2014. "Synthesis, Characterisation and Antimicrobial Activity of Bis(Phthalazine-1-Hydrazone)-2,6-Diacetylpyridine and Its Complexes with CoIII, NiII, CuII and ZnII." Polyhedron 80:142-50.

Mosmann, T. 1983. "Rapid Colorimetric Assay for Cellular Growth and Survival: Application to Proliferation and Cytotoxicity Assays." Journal of Immunological Methods 65(1-2):55-63.

Olmo, Esther del, Bianca Barboza, Ma Inés Ybarra, José Luis López-Pérez, Rosalía Carrón, Ma Angeles Sevilla, Cinthia Boselli, and Arturo San Feliciano. 2006. "Vasorelaxant Activity of Phthalazinones and Related Compounds." Bioorganic \& Medicinal Chemistry Letters 16(10):2786-90.

Sun, Xian-Yu, Chuan Hu, Xian-Qing Deng, Cheng-Xi Wei, Zhi-Gang Sun, and ZheShan Quan. 2010. "Synthesis and Anti-Inflammatory Activity Evaluation of Some Novel 6-Alkoxy(Phenoxy)-[1,2,4]Triazolo[3,4-a]Phthalazine-3-Amine Derivatives." European Journal of Medicinal Chemistry 45(11):4807-12.

Sun, Xian-Yu, Cheng-Xi Wei, Xian-Qing Deng, Zhi-Gang Sun, and Zhe-Shan Quan. 2010. "Evaluation of the Anticonvulsant Activity of 6-(4-Chlorophenyoxy)Tetrazolo[5,1-a]Phthalazine in Various Experimental Seizure Models in Mice." 
Pharmacological Reports 62(2):273-77.

Sun, Xian-Yu, Cheng-Xi Wei, Xian-Qing Deng, Zhi-Gang Sun, and Zhe-Shan Quan. 2011. "Synthesis and Primary Anticonvulsant Activity Evaluation of 6-AlkyoxylTetrazolo[5,1-a]Phthalazine Derivatives.” Arzneimittelforschung 60(06):289-92.

Turky, Abdallah, Ashraf H. Bayoumi, Farag F. Sherbiny, Khaled El-Adl, and Hamada S. Abulkhair. 2020. "Unravelling the Anticancer Potency of 1,2,4-TriazoleN-Arylamide Hybrids through Inhibition of STAT3: Synthesis and in Silico Mechanistic Studies." Molecular Diversity. 25(1), 403-420.

Turky, Abdallah, Farag F. Sherbiny, Ashraf Bayoumi, Hany E. A. Ahmed, and Hamada S. Abulkhair. 2020. "Novel 1,2,4-triazole Derivatives: Design, Synthesis, Anticancer Evaluation, Molecular Docking, and Pharmacokinetic Profiling Studies." Archiv Der Pharmazie, 353(12),2000170.

Wood E. R., Truesdale A. T., McDonald O. B., Yuan, D., Hassell A., Dickerson, S. H \& Shewchuk L. 2004. "A unique structure for epidermal growth factor receptor bound to GW572016 (Lapatinib): relationships among protein conformation, inhibitor off-rate, and receptor activity in tumor cells". Cancer research, 64(18), 6652-6659.

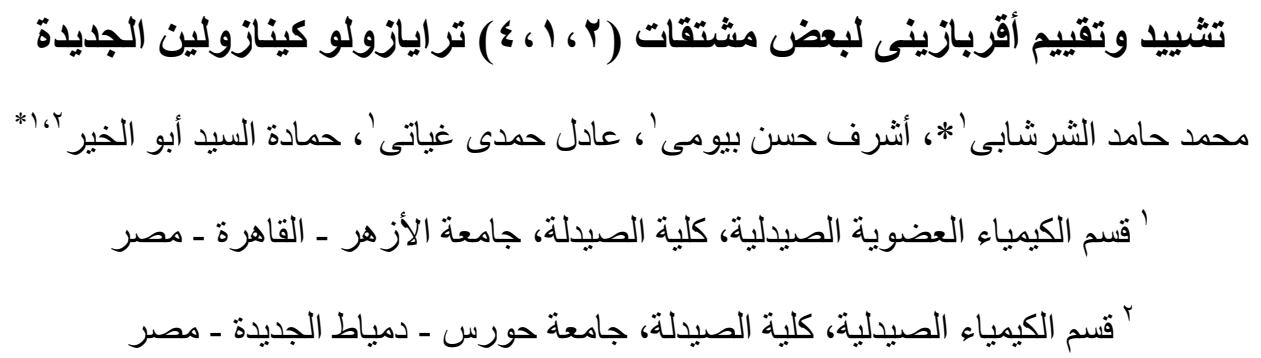

hamadaorganic@azhar.edu.eg البريد الإليكترونى للباحث الرئيسى

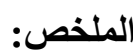

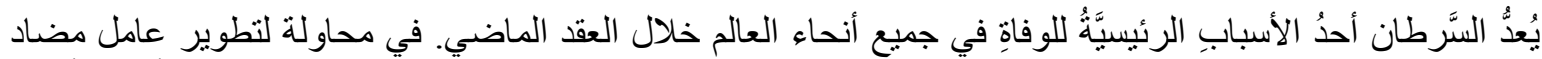

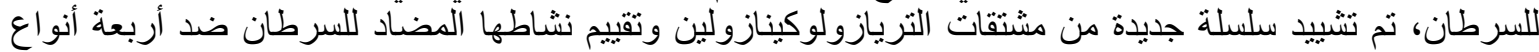

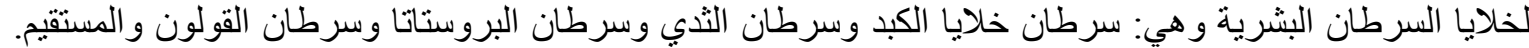

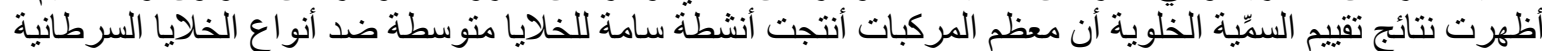

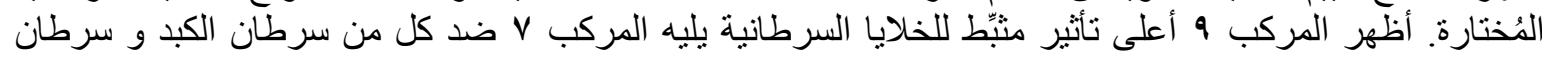

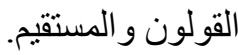

